\title{
LOCATION OF MULTIPLE SERVER COMMON SERVICE CENTERS OR PUBLIC FACILITIES FOR MINIMIZING GENERAL CONGESTION AND TRAVEL COST FUNCTIONS
}

\author{
V. Marianov ${ }^{1}$, D. Serra ${ }^{2}$ \\ ${ }^{1}$ Department of Electrical Engineering \\ Pontificia Universidad Católica de Chile, Santiago, CHILE \\ e-mail:marianov@ing.puc.cl \\ ${ }^{2}$ Department of Economics and Business, and GREL-IET \\ Universitat Pompeu Fabra, Barcelona, SPAIN \\ e-mail:daniel.serra@econ.upf.es
}

\begin{abstract}
We propose a model and solution methods, for locating a fixed number of multiple-server, congestible common service centers or congestible public facilities. Locations are chosen so to minimize consumers' congestion (or queuing) and travel costs, considering that all the demand must be served. Customers choose the facilities to which they travel in order to receive service at minimum travel and congestion cost. As a proxy for this criterion, total travel and waiting costs are minimized. The travel cost is a general function of the origin and destination of the demand, while the congestion cost is a general function of the number of customers in queue at the facilities.
\end{abstract}

Keywords: Location modelling, congestion costs

AMS Classification: 90B22, 90B15, 90B80

JEL Classification: C61, J80 


\section{Introduction and literature review}

Some large organizations, with geographically distributed or extensive facilities, have internal clients (workers) that must receive some service at centers belonging to the same company. These centers must be located in such a way that people working at any facility can use them; workers have to travel from their workplaces to these centers and wait until served. In this case, the companies may want to minimize the cost associated with the time spent by their workers both traveling to, and waiting in lines at these centers. An instance of this setting is a large university campus with many buildings, where food or similar services need to be located to serve all workers and students. The same situation occurs when there are common service centers established as joint ventures by their users. Examples are the co-op stores; the users want to have the centers as close as possible to them, and to receive a fast service. Otherwise, the benefit of having established the common centers or stores may not be clear to some of them. More examples can be found among public services, electrical utilities, phone companies, and so on, with geographically distributed offices. Although the total demand for attention at these offices does not depend on the locations or number of service centers, the providers may wish to promote a good image, by minimizing customers' inconvenience (or cost) related to travel and waiting time. In turn, each individual user or customer minimizes her/his own travel and waiting costs when seeking service. Assuming the customers of the services under study are a small part of all the people traveling over the network, travel costs depend only on the distance or the time it takes to the customer to go from her/his starting point to the center she/he chooses. However, waiting costs depend jointly on the decisions made by all customers. In fact, the more customers decide to patronize some center, the more congested it becomes, so the waiting time of all the customers using this center increases. Furthermore, the cost that customers assign to the time they spend traveling may be very different to the cost they assign to the time spent waiting at the center. Also, different types of services can have different cost functions associated to travel and waiting. Finally, different customers could potentially have different perceptions of the cost of time (cost functions).

All these facts must be taken into account when the company, service or group of users have to decide on how many centers to open, where to locate them, and how many servers (clerks, attendants, cashiers, machines) to allocate to each one of them, so to minimize the travel and waiting cost functions. We propose a method for making these decisions.

A very good review of published models, as well as some additional models for location on congested networks, is presented in Brandeau et al (1995). The models cover several types of demand (elastic or inelastic to congestion and travel time) and assignment of customers to facilities (either forced by a central planner or made by user choice). In most cases of inelastic demand, the models locate one, or at most two facilities. In all cases, additional to travel distance or time, the queuing delay is the main issue, as opposed to the number of 
customers in the system. As we will show later, formulas of queuing delay are more difficult to handle than formulas of number of customers in the system, although delay and number of customers are related and consequently, are equivalent.

Lately, additional models for location of congested facilities have been published. Marianov and Serra $(1998,2001)$, rather than minimizing waiting time, choose to use a constraint that forces the probability of a queue longer than a certain number, to be less than a predefined value. Jamil et al (1999) locate a single server, as opposed to several, multiple-server facilities. In the area of telecommunications, many approaches have appeared in the literature for the design of networks so as to either minimize communications delay or to keep this delay constrained to some extent (Rolland et al, 1999). Although these models are similar to those used for facility location, again, they consider queuing delay in their formulations.

The model we propose optimizes the location of a fixed number of service centers, each with several servers, in such a way that the total travel and waiting costs are minimized, as two different objectives. The number of servers per center is fixed, but can be determined by repeatedly solving the model. As opposed to existing models, in the model we present, instead of minimizing the travel and expected waiting time, we minimize separately the travel cost and the expected waiting cost, computed as an expected cost, averaged over the number of customers in the system. We also allow the possibility that for customers coming from different origins, the cost function might be different (although we do not provide a solution method for this case); thus, we can take into account the differences in customers' perceptions when optimizing the quality of service of the whole system. Furthermore, we provide formulations that allow us to use general cost functions, which in the case of congestion is done through the use of queue lengths instead of waiting times, being both equally meaningful measures of congestion.

If both cost objectives are measured in monetary terms, they can be added to obtain a single objective. Nevertheless, if their units are different, for example, travel and waiting times instead of travel and waiting costs, which can be perceived differently by customers, then a multiobjective approach has to be chosen. Furthermore, one of the objectives is nonlinear.

We present the model together with two solution procedures. The first procedure solves the problem if objectives are cast in monetary terms, or if the weighting method is chosen for the multi-objective optimization formulation. This procedure uses a metaheuristic based on tabu search and ant-colonies to solve the problem.

The second procedure solves the multi-objective, nonlinear optimization model, using the constraint method (Cohon, 1978). In this method, one of the objectives is kept as such, while the remaining objectives are constrained to have acceptable values. These acceptable values or right hand sides of the new constraints, take different values in successive runs, so that trade-off curves can be obtained. In our case, by transforming the nonlinear objective into a constraint, we are also able to linearize the problem and solve it using commercial integer programming software, provided some simplifying assumptions are made. 
The paper is structured as follows. We first develop the nonlinear multiobjective formulation of the problem. Then we present the metaheuristic which is useful when the weighting method is chosen, or when both objectives are measured in monetary terms and can be added to form a single objective. We offer computational experience on a set of test instances of the problem. Then, we present the constraint method, and provide some computational experience on a 55-node network using CPLEX. We do not use the same instances of the problem for both methods, because in this way the reader can see different interesting aspects of the problem and the methods.

\section{Development of the multiple-server model}

The formulation is cast using $p$-median constraints. The model locates $p$ facilities, each with $s$ servers, and determines the allocation of the demands to each facility, so that the travel and waiting cost functions are minimized, as follows:

$$
\begin{array}{ll}
\text { Min } & {\left[\mathrm{Z}_{1}, \mathrm{Z}_{2}\right]} \\
& \mathrm{Z}_{1}=(\text { Travel Cost }) \\
& \mathrm{Z}_{2}=(\text { Expected Congestion Cost }) \\
\text { s. t. } & \sum_{j} x_{i j}=1 \quad \forall i \\
& x_{i j} \leq y_{j} \quad \forall i, j \\
& \sum_{j} y_{j}=p \\
& x_{i j} \in\{0,1\} \quad \forall i, j \\
& y_{j} \in\{0,1\} \quad \forall j
\end{array}
$$

In this model, variable $x_{i j}$ is one if demand $i$ is allocated to facility $j$, and zero otherwise. Variable $y_{j}$ is one when a facility is located at candidate site $j$, and zero otherwise. The objectives to be minimized are both costs. Constraint (2) forces all demands to be allocated to exactly one facility. Constraint (3) states that a demand cannot be served from a site without a facility. Constraint (4) forces $p$ facilities to be located, and constraint (5) ensures integrality of the variables.

We now propose expressions for the cost functions.

\section{$\underline{\text { Travel Cost Function }}$}

Finding an expression for a general travel cost function is done in the same way as in the $p$ median-like models. The travel cost per customer is a function of the customer's origin (demand node) and destination (facility), or travel distance, or travel time. This function 
can take any form. It could be nonlinear, discontinuous and non-differentiable. It could be even a different function for each pair origin - destination. Each origin (demand node) $i$ is known, and for each origin there are a finite number of destinations $j$. Thus, the value $c_{i j}$ of this general function can be computed for each pair origin-destination $(i, j)$ before solving the optimization problem, and this value $c_{i j}$ used in the first objective as follows:

$$
Z_{1}=\sum_{i} \sum_{j} h_{i} c_{i j} x_{i j}
$$

where $c_{i j}$ is the cost (per customer) of traveling from $i$ to $j$, and $h_{i}$ is the demand rate at demand node $i$.

\section{Congestion Costs}

In most of the existing models, congestion is expressed in terms of the expected waiting times (or delays). In order to obtain a tractable mathematical function representing a general cost function to be minimized, we use the number of customers in the system instead, and minimize directly an expected cost. Recall that the expected waiting time and the expected number of customers in the system are related through Little's law, so both are equivalent. In order to compute the expected congestion cost, we assume a finite queue multiple servers $\mathrm{M} / \mathrm{M} / \mathrm{s} / \mathrm{K}$ queuing system at each facility. Calls arrive to each facility at a rate $\lambda_{j}$, given by:

$$
\lambda_{j}=\sum_{i} h_{i} x_{i j}
$$

and are served at a rate $\mu_{j}$. Each state $n$ of the queuing system, representing $n$ customers in the system, has a probability $P_{n}\left(\lambda_{j}\right)$, which depends on the arrival rate $\lambda_{j}$. Equivalently, if $\rho_{j}=\lambda_{j} / \mu_{j} \leq s$, and $s$ is the number of servers at $j$, the probability can be expressed as $P_{n}\left(\rho_{j}\right)$, according to the following equations (Hillier and Lieberman, 1986), where the subscript $j$ has been omitted for simplicity purposes:

$$
\begin{aligned}
& P_{0}(\rho)=\left[1+\sum_{n=1}^{s} \frac{\rho^{n}}{n !}+\frac{\rho^{s}}{s !} \sum_{n=s+1}^{K}\left(\frac{\rho}{s}\right)^{n-s}\right]^{-1} \\
& P_{n}(\rho)=\left\{\begin{array}{cc}
\frac{\rho^{n}}{n !} P_{0}(\rho) & \text { for } n \leq s \\
\frac{\rho^{n}}{s ! s^{n-s}} P_{0}(\rho) & \text { for } s \leq n \leq K \\
0 & \text { for } n \geq K
\end{array}\right.
\end{aligned}
$$


One set of similar equations can be written for each facility $j$. Instead of using explicitly a function of the waiting time in the objective, we assume that for a customer originating at node $i$, her/his congestion cost function (possibly different for each $i$ ) is discrete, and depends on the number $n$ of customers (including him/herself) at the facility she/he chooses. This value is $b_{i n}$. Using this value and the probabilities just defined, the expected queuing cost at a facility $j$ for a customer originating at $i$ is:

$$
\sum_{n=0}^{K} b_{i n} P_{n}\left(\lambda_{j}\right) \text {. }
$$

For all the customers patronizing the facility $j$, the total cost is:

$$
\sum_{i} h_{i} x_{i j} \sum_{n=0}^{K} b_{i n} P_{n}\left(\lambda_{j}\right)=\sum_{i} \sum_{n=0}^{K} h_{i} x_{i j} b_{i n} P_{n}\left(\sum_{i} h_{i} x_{i j}\right)
$$

Then, the objectives of the model are:

$$
\begin{aligned}
& \mathrm{Z}_{1}=\sum_{i} \sum_{j} h_{i} c_{i j} x_{i j} \text { and } \\
& \mathrm{Z}_{2}=\sum_{j} \sum_{i} \sum_{n=0}^{K} h_{i} x_{i j} b_{i n} P_{n}\left(\sum_{i} h_{i} x_{i j}\right)
\end{aligned}
$$

Adding constraints (2) to (5) completes the model. Note that objective $Z_{2}$ is the sum of the costs over all facilities $j$. The second objective is nonlinear.

\section{A metaheuristic for the non-linear integer formulation}

The model presented in the previous section is a combinatorial optimization problem. Many combinatorial problems are intractable and belong to the class of NP-Hard problems. Kariv and Hakimi (1979) prove that the p-Median problem is an NP-Hard problem on a general graph. Moreover, in this case, the inclusion of a non-linear additional objective reinforces the difficulty of solving the problem optimally.

The common belief in this field is that no efficient algorithm could ever be found to solve these inherently hard problems. Heuristics, and recently metaheuristics, are considered one of the choice methods for solving hard combination optimization problems. We propose the use of a metaheuristic procedure based on the MAX-MIN ant system together with TABU search for finding solutions to the proposed formulation.

The Ant System introduced by Colorni et al (1991a, 1991b), Dorigo et al (1996), Dorigo and Di Caro (1999), is a cooperative search algorithm inspired by the behavior of real ants. Ants lay down some quantity of an aromatic substance, known as pheromone, on their way to food. The likelihood with which an ant chooses a specific path, is correlated with the intensity of the pheromone on that path. The pheromone trail evaporates over time if no more pheromone is laid down by other ants, therefore the best paths have more intense 
pheromone and higher probability of being chosen. The Ant System approach associates pheromone trails to features of the solutions of a combinatorial problem, and can be seen as a kind of adaptive memory of previous solutions. Solutions are iteratively constructed in a randomized heuristic fashion biased by the pheromone trails left by the previous ants. The pheromone trails, $\tau_{j}$, are updated after the construction of a solution, ensuring that the best features will have a more intense pheromone.

Recently, Stützle (1997) have proposed an improved version of the Ant System, denominated MAX-MIN Ant System. The MAX-MIN Ant system differs from the Ant System in the following way: only the best ant updates the trails in every cycle. To avoid stagnation of the search, i.e. ants always choosing the same path, Stüzle (1998a) proposed a lower and upper limit to the pheromone trail, $\tau_{\min }$ and $\tau_{\max }$, respectively. Stützle and Hoos (1999), Stützle $(1997,1998$ a) applied this procedure to the Traveling Salesman Problem, The Quadratic Assignment Problem and The Flow-Shop Scheduling Problem; and Lourenço and Serra (2000) applied it to the Generalized Assignment Problem.

Tabu Search is a metaheuristic that guides local heuristic search procedures to explore the solution space beyond local optimality. It was introduced by Glover $(1989,1990)$ and, in essence, Tabu Search explores a part of the solution space by repeatedly examining all neighborhoods of the current solution, and moving to the best neighborhood even if this leads to a deterioration of the objective function. This approach tries to avoid being trapped in a local optimum. In order to avoid the cycling back to a solution that has recently been examined, solutions are inserted in a tabu list that is constantly updated. Additionally, several criteria of flexibility can be used in the tabu search including aspiration and diversification.

The aspiration criterion is used as an insurance against restricting moves which would have led to finding high quality solutions. In other words, the aspiration criteria determines when a move to a new solution is done, even if tabu. Usually, this criterion states that if a move produces a feasible solution that is better than the best known solution, then the tabu status is disregarded and the move is executed. The diversification criterion is utilized to escape from local optima and is achieved by using a long - term memory function. It allows a broader exploration of the solution space by starting from solutions that have not been well explored. A more detailed description can be found in Appendix 1.

This method has been successfully applied to a wide variety of location problems: p-hub Location Problems (Klincewicz, 1992) and (Marianov et al, 1999), (r $\mid$ Xp)- Medianoid and ( $\mathrm{r} \mid p$ )- Centroid Problems (Benati and Laporte, 1994), the Vehicle Routing Problem (Gendreau et al, 1994) and p-Median Problem (Rolland et al, 1996).

In order to use the MAX-MIN ant system, we define the pheromone trail $\tau_{j}$ as the desirability of locating a center in $j$. The higher $\tau_{j}$ is, the more desirable is the location of a center in that node. The $\tau_{j}$ are initially set as 


$$
\tau_{j}=\left[\sum_{i} h_{i} c_{i j}\right]^{-1},
$$

that is the inverse of the weighted travel cost from all demand nodes to the facility. In essence, once the initial $\tau_{j}$ are computed, the procedure has the following steps:

1. Construction of an initial solution. First, the nodes are ordered with respect to the probability function defined by

$$
p_{j}=\frac{\tau_{j}}{\sum_{l \in J} \tau_{l}}
$$

The initial solution is chosen randomly, taking into account the probability distribution previously defined.

2. Solutions in the neighborhood of the initial solution are searched in an attempt to improve the initial solution. Tabu search is applied.

3. The pheromone trails are updated using the current solution in the following way: $\tau_{j}^{\text {new }}=\rho \tau_{j}^{\text {old }}+\Delta \tau_{j}$, where $\rho(0<\rho<1)$ is the persistence of the trail, i.e. $1-\rho$, represents the evaporation. The updated amount is

$$
\Delta \tau_{j}= \begin{cases}\tau_{\max } Q & \text { If an outlet is located at } \mathrm{j} \\ 0, & \text { otherwise }\end{cases}
$$

where $Q$ is a preset parameter $(Q<1)$.

4. In the final stage, the MAX-MIN limits are checked and imposed $\tau_{\min } \leq \tau_{j} \leq \tau_{\max } \forall j$ $\in J$, if the updated pheromone falls outside the interval.

The termination condition of this iterative procedure is the number of total iterations. A formal description of the metaheuristic procedure is presented in Appendix 1.

\section{Computational Experience with the metaheuristic procedure}

The non-linear formulation has been tested as follows: several random networks with 30 and 50 nodes $(n)$ were generated. For each network, the demand rate at each node was randomly set between 100 and 500. Congestion costs were set equal for all customers regardless of location. If $j$ represents the number of people in queue, these cost where set as follows: $c_{j}=s / 10$ for $j \leq s$ and $c_{j}=c(j-1)+1 / 10$ for $j>s$. Also, for each network, the number of facilities $(p)$ to be located was set to 3, 4 and 5 and for each $n$ and $p$ the number of servers $(s)$ was set to 2,3 and 4 . The service rate was set equal to 


$$
\frac{\text { fact } * \text { calltot }}{p}
$$

where calltot is the total number of calls in the system, $p$ the number of facilities to be located and fact is a preset factor equal to 2, 3 and 4 .

For each $n, p, s$ and fact, 25 networks were generated, giving a total of 1,350 instances of the metaheuristic. The maximum number of people in the queue was set to 10 .

For each instance of the metaheuristic, the number of iterations in the Max-min ant-colony procedure was set to 50 . For each instance of the tabu search heuristic, the number of oneopt trades in the tabu phase of the heuristic was set to 40 . The diversification phase was executed three times. The size of the neighborhood (the $m$ nodes around the current solution) was set between 4 and 8 . The size of the tabu list was set to 6 . The values of the parameters of the metaheuristic were set to $Q=0.10, \rho=0.75, \tau_{\max }=2 p * \max \left(\tau_{j}\right)$ and $\tau_{\min }=(1 / 2 p) * \min \left(\tau_{j}\right)$ (where, $p$ is the number of centers to locate).

In order to check the results obtained by the metaheuristic, the 1,350 instances were also solved using complete enumeration. Results are presented in Tables 1 and 2. For both tables, the first column (NP) represents de number of facilities to be located. In the second column (S) the number of servers is listed. The third column indicates the preset factor used to compute the service rate. The fourth and fifth colums represent de number of instances were the algorithm did not obtain optimal solutions in total and percentage terms respectively. In the sixth and seventh columns the average and maximum deviation from optimality is presented. Finally, the last column shows the average computer times in seconds.

For $n=30,94.6 \%$ of the 675 results obtained with the metaheuristic were optimal. The average deviation from optimality was equal to $0.97 \%$ and the maximum deviation $(4.10 \%)$ was obtained when $p=2, s=4$, and fact $=3$. The average computing time ranged between 4.79 and 10.42 seconds on a Pentium III with $256 \mathrm{Mb}$ of RAM.

For $n=50,94.5 \%$ of the 675 results obtained with the metaheuristic were optimal. The average deviation from optimality was equal to $1,46 \%$ and the maximum deviation $(9.63 \%)$ was obtained when $p=2, s=3$, and fact $=2$. The average computing time ranged between 7.61 and 16.77 seconds. 


\begin{tabular}{|c|c|c|c|c|c|c|c|}
\hline NP & $\mathrm{S}$ & FAC & $\begin{array}{c}\text { Nonoptimal } \\
\text { solutions }\end{array}$ & $\begin{array}{l}\text { \% non } \\
\text { optimal }\end{array}$ & $\begin{array}{l}\text { Average } \\
\text { deviation }\end{array}$ & $\begin{array}{c}\text { Maximum } \\
\text { deviation }\end{array}$ & $\begin{array}{l}\text { Average } \\
\text { time }\end{array}$ \\
\hline \multirow{9}{*}{3} & \multirow{3}{*}{2} & 2 & 0 & \multirow{3}{*}{$1.3 \%$} & - & - & 4.77 \\
\hline & & 3 & 0 & & - & - & 4.74 \\
\hline & & 4 & 1 & & $0.41 \%$ & $0.41 \%$ & 4.69 \\
\hline & \multirow{3}{*}{3} & 2 & 2 & \multirow{3}{*}{$4.0 \%$} & $1.37 \%$ & $1.85 \%$ & 4.87 \\
\hline & & 3 & 1 & & $2.35 \%$ & $2.35 \%$ & 4.83 \\
\hline & & 4 & 0 & & - & - & 4.76 \\
\hline & \multirow{3}{*}{4} & 2 & 1 & \multirow{3}{*}{$1.3 \%$} & $1.52 \%$ & $1.52 \%$ & 4.75 \\
\hline & & 3 & 0 & & - & - & 4.75 \\
\hline & & 4 & 0 & & - & - & 4.88 \\
\hline \multirow{9}{*}{4} & \multirow{3}{*}{2} & 2 & 3 & \multirow{3}{*}{$8.0 \%$} & $1.69 \%$ & $3.17 \%$ & 7.18 \\
\hline & & 3 & 2 & & $1.41 \%$ & $2.88 \%$ & 7.17 \\
\hline & & 4 & 1 & & $0.63 \%$ & $0.63 \%$ & 7.26 \\
\hline & \multirow{3}{*}{3} & 2 & 3 & \multirow{3}{*}{$8.0 \%$} & $1.42 \%$ & $3.24 \%$ & 7.27 \\
\hline & & 3 & 2 & & $1.14 \%$ & $3.00 \%$ & 7.50 \\
\hline & & 4 & 1 & & $0.97 \%$ & $1.02 \%$ & 7.49 \\
\hline & \multirow{3}{*}{4} & 2 & 2 & \multirow{3}{*}{$6.6 \%$} & $1.32 \%$ & $3.84 \%$ & 7.38 \\
\hline & & 3 & 1 & & $0.13 \%$ & $1.13 \%$ & 7.26 \\
\hline & & 4 & 2 & & $1.72 \%$ & $3.74 \%$ & 7.30 \\
\hline \multirow{9}{*}{5} & \multirow{3}{*}{2} & 2 & 0 & \multirow{3}{*}{$6.6 \%$} & $1.85 \%$ & $2.21 \%$ & 10.17 \\
\hline & & 3 & 2 & & $1.19 \%$ & $2.97 \%$ & 9.93 \\
\hline & & 4 & 3 & & $1.80 \%$ & $4.10 \%$ & 9.95 \\
\hline & \multirow{3}{*}{3} & 2 & 1 & \multirow{3}{*}{$5.3 \%$} & $1.12 \%$ & $1.66 \%$ & 10.38 \\
\hline & & 3 & 1 & & $0.65 \%$ & $0.90 \%$ & 10.34 \\
\hline & & 4 & 2 & & $1.07 \%$ & $2.28 \%$ & 10.34 \\
\hline & \multirow{3}{*}{4} & 2 & 1 & \multirow{3}{*}{$6.0 \%$} & $0.15 \%$ & $0.39 \%$ & 10.39 \\
\hline & & 3 & 2 & & $1.37 \%$ & $2.09 \%$ & 10.42 \\
\hline & & 4 & 3 & & $1.16 \%$ & $3.65 \%$ & 10.17 \\
\hline
\end{tabular}

Table 1: Results with $n=30$ 


\begin{tabular}{|c|c|c|c|c|c|c|c|}
\hline NP & $\mathrm{S}$ & FAC & $\begin{array}{c}\text { Nonoptimal } \\
\text { solutions }\end{array}$ & $\begin{array}{l}\% \text { non } \\
\text { optimal }\end{array}$ & $\begin{array}{l}\text { Average } \\
\text { deviation }\end{array}$ & $\begin{array}{c}\text { Maximum } \\
\text { deviation }\end{array}$ & $\begin{array}{c}\text { Average } \\
\text { time }\end{array}$ \\
\hline \multirow{9}{*}{3} & \multirow{3}{*}{2} & 2 & 1 & \multirow{3}{*}{$5.3 \%$} & $0.25 \%$ & $0.25 \%$ & 7.67 \\
\hline & & 3 & 2 & & $1.26 \%$ & $2.44 \%$ & 7.61 \\
\hline & & 4 & 0 & & - & - & 7.67 \\
\hline & \multirow{3}{*}{3} & 2 & 0 & \multirow{3}{*}{$4.0 \%$} & - & - & 7.76 \\
\hline & & 3 & 1 & & $0.35 \%$ & $0.35 \%$ & 7.80 \\
\hline & & 4 & 2 & & $1.28 \%$ & $2.09 \%$ & 7.72 \\
\hline & \multirow{3}{*}{4} & 2 & 0 & \multirow{3}{*}{$2.6 \%$} & - & - & 7.87 \\
\hline & & 3 & 0 & & - & - & 7.70 \\
\hline & & 4 & 2 & & $1.54 \%$ & $1.92 \%$ & 7.80 \\
\hline \multirow{9}{*}{4} & \multirow{3}{*}{2} & 2 & 1 & \multirow{3}{*}{$8.0 \%$} & $1.07 \%$ & $3.86 \%$ & 11.52 \\
\hline & & 3 & 2 & & $1.59 \%$ & $5.17 \%$ & 11.65 \\
\hline & & 4 & 3 & & $1.95 \%$ & $3.19 \%$ & 11.57 \\
\hline & \multirow{3}{*}{3} & 2 & 1 & \multirow{3}{*}{$6.6 \%$} & $1.62 \%$ & $1.82 \%$ & 11.82 \\
\hline & & 3 & 1 & & $2.67 \%$ & $6.66 \%$ & 11.92 \\
\hline & & 4 & 3 & & $1.32 \%$ & $3.23 \%$ & 11.92 \\
\hline & \multirow{3}{*}{4} & 2 & 2 & \multirow{3}{*}{$10.6 \%$} & $1.00 \%$ & $2.92 \%$ & 11.84 \\
\hline & & 3 & 3 & & $2.39 \%$ & $2.88 \%$ & 11.96 \\
\hline & & 4 & 3 & & $0.21 \%$ & $0.36 \%$ & 11.70 \\
\hline \multirow{9}{*}{5} & \multirow{3}{*}{2} & 2 & 4 & \multirow{3}{*}{$9.3 \%$} & $2.09 \%$ & $4.48 \%$ & 16.45 \\
\hline & & 3 & 2 & & $4.86 \%$ & $9.63 \%$ & 16.44 \\
\hline & & 4 & 1 & & $3.99 \%$ & $6.70 \%$ & 16.50 \\
\hline & \multirow{3}{*}{3} & 2 & 3 & \multirow{3}{*}{$14.6 \%$} & $1.12 \%$ & $2.36 \%$ & 16.77 \\
\hline & & 3 & 4 & & $1.96 \%$ & $5.47 \%$ & 16.55 \\
\hline & & 4 & 5 & & $1.50 \%$ & $4.62 \%$ & 13.75 \\
\hline & \multirow{3}{*}{4} & 2 & 2 & \multirow{3}{*}{$13.3 \%$} & $1.89 \%$ & $3.78 \%$ & 13.88 \\
\hline & & 3 & 4 & & $2.02 \%$ & $5.03 \%$ & 13.86 \\
\hline & & 4 & 4 & & $2.57 \%$ & $6.56 \%$ & 13.96 \\
\hline
\end{tabular}

Table 2: Results with $n=50$ 


\section{The Constraint Method}

\section{Linearization of the model}

As noted before, objective $Z_{2}$ is nonlinear. However, under some conditions on the cost factors $b_{i n}$, if the multiobjective problem is solved using the constraint method, and if objective $Z_{2}$ is disaggregated by facilities, the problem can be linearized.

The constraint method consists in constraining $(m-1)$ of the $m$ objectives of the problem to have "acceptable" values. The remaining objective is optimized, subject to the original constraints of the problem, plus the extra $(m-1)$ new constraints. The "acceptable" values are modified and the problem solved again, as many times as needed for obtaining adequate trade-off curves. In our case, the model has two objectives to be minimized. The first objective is left as such (the travel costs objective), while the nonlinear congestion costs objective is disaggregated by facilities and each component constrained to have a value of at most $C_{j}$. This value is modified in successive runs of the model, and a trade-off curve is drawn.

The model becomes:

$$
\operatorname{Min} \sum_{i} \sum_{j} h_{i} c_{i j} x_{i j}
$$

Subject to

$$
\sum_{i} \sum_{n=0}^{K} h_{i} x_{i j} b_{i n} P_{n}\left(\sum_{i} h_{i} x_{i j}\right) \leq C_{j} \quad \forall j
$$

and subject to (2) to (5).

This version of the model has a linear objective and linear constraints, except for constraint (11). In order to linearize this constraint, we first note that $b_{\text {in }}$ is always increasing (at least, non-decreasing) with $n$, because customers' waiting cost is increasing with the number of customers in the system (except for crowd - loving customers, case we are not including here). If, additionally, the waiting cost $b_{\text {in }}$ does not depend on the origin of the customers, that is $b_{i n}=b_{n}$ for all $i$, then the constraint can be written as:

$$
\left(\sum_{i} h_{i} x_{i j}\right)\left(\sum_{n=0}^{K} b_{n} P_{n}\left(\sum_{i} h_{i} x_{i j}\right)\right) \leq C_{j} \quad \forall j
$$

The left hand side of constraint (12) strictly increases with $\sum_{i} h_{i} x_{i j}$ (or $\lambda_{j}$ ), which we prove next. 
Suppose for the moment that $b_{n}=1$ for all $n$. The second factor on the left hand side of constraint (12) becomes $\sum_{n=0}^{K} P_{n}\left(\sum_{u} h_{i} x_{i j}\right)$. This expression is always equal to one, for all values of $\sum_{i} h_{i} x_{i j}$, because it is just the sum of all probabilities of numbers of customers in the queuing system. When $\sum_{i} h_{i} x_{i j}$ increases, the probabilities of more customers in the system (larger values of $n$ ) must increase. The probabilities of fewer customers in the system (smaller values of $n$ ) must then decrease in the same amount, to keep the total probability sum equal to one.

We now relax the requirement that $b_{n}=1$, and recall that $b_{n}$ is non-decreasing with $n$. Since higher order probabilities are multiplied by numbers $b_{n}$ that are larger (or at least, equal), $\sum_{n=0}^{K} b_{n} P_{n}\left(\sum_{i} h_{i} x_{i j}\right)$ is non-decreasing with $\sum_{i} h_{i} x_{i j}$. Since in constraint (12), this nondecreasing expression is multiplied by the strictly increasing expression $\left(\sum_{i} h_{i} x_{i j}\right)$, it is easy to see that the product is strictly increasing, which completes the proof.

As constraint (12) is strictly increasing with $\sum_{i} h_{i} x_{i j}$, there must exist a number $\hat{C}_{j}$, such that whenever:

$$
\sum_{i} h_{i} x_{i j} \leq \hat{C}_{j} \quad \forall j
$$

constraint (12) necessarily holds true. Consequently, equation (13) is a linear expression that can be used instead of constraint (12) in the optimization formulation. For each value of $C_{j}$ in (12), the value of $\hat{C}_{j}$ in the equivalent linear constraint (13) is found by computing the value of $\sum_{i} h_{i} x_{i j}$ for which equation (12) holds as equality.

The final, linear model for the case $b_{i n}=b_{n}$ for all $i$, is:

$$
\begin{array}{ll}
\text { Min } & \sum_{i} \sum_{j} h_{i} c_{i j} x_{i j} \\
\text { s.t. } & \sum_{j} x_{i j}=1 \quad \forall i \\
& x_{i j} \leq y_{j} \quad \forall i, j \\
& \sum_{j} y_{j}=p
\end{array}
$$




$$
\begin{array}{lc}
\sum_{i} h_{i} x_{i j} \leq \hat{C}_{j} & \forall j . \\
x_{i j} \in\{0,1\} & \forall i, j \\
y_{j} \in\{0,1\} & \forall j
\end{array}
$$

\section{Solution, analysis and calibration of the number s of servers per facility}

The usual constraint method uses Branch and Bound (or any feasible solution method for integer programming formulations) and the following steps:

1. Determine the values of the cost parameters $\left(c_{i j}\right.$ and $\left.b_{n}\right)$. These values can be found from polls or focus groups. Set values for $C_{j}, s, K$ and $\mu_{j}$. Note that potentially, $s$ and $K$ can be also facility-dependent.

2. Starting from the values of $C_{j}$ and remaining parameters, compute the value of $\sum_{i} h_{i} x_{i j}$ for which equation (12) holds as equality. Set $\hat{C}_{j}$ to this value.

3. Solve the model.

4. Modify the value of $C_{j}$ using an adequate step size and direction. Go to step 2 until a sufficiently detailed trade off curve can be drawn.

This procedure would provide the necessary trade-off curves. However, a deeper analysis can be performed, which is useful for the determination of some parameters while doing the design of the system. Furthermore, in the absence of reliable information on waiting cost functions, this analysis shows the effects of using different cost function shapes.

Note first that a particular value of the parameter $\hat{C}_{j}$ in equation (13) could be the result of more than one combination of values of the relevant parameters, namely service rate $\mu_{j}$, maximum queue length $K_{j}$, number of servers in each center $s_{j}$, and queuing cost function $\left\{b_{n}\right\}$. Once $\hat{C}_{j}$ is computed and plugged in constraint (13), the particular combination of values that were used for its computation becomes unimportant from the point of view of the optimization problem, which becomes a capacitated $p$-median. Furthermore, the only relevant figures of the problem at this stage are the parameters $\hat{C}_{j}$ and $p$, as well as the demand rates $h_{i}$ at each node and travel $\operatorname{costs} c_{i j}$ between demand nodes and potential center sites. Then, we can separately perform the following stages:

Stage 1 . Using the known data $\left(h_{i}, c_{i j}\right)$, solve the capacitated linear integer optimization problem, for all required values of $p$ and using the full range of possible values of $\hat{C}_{j}$. As a result of this stage, for each $p$, there is a set of solutions. Each solution 
corresponds to a value of the travel cost objective, and the demand figures at each facility, for that value of the objective. In order to determine the full range, of relevant values of $\hat{C}_{j}$, needed to perform this stage, note that if we add both sides of constraint (13) over all possible location candidate nodes, we obtain

$$
\begin{aligned}
& \sum_{i} h_{i} \sum_{j} x_{i j} \leq \sum_{j} \hat{C}_{j}, \text { or } \\
& \sum_{i} h_{i} \leq \sum_{j} \hat{C}_{j} .
\end{aligned}
$$

The left hand side of this expression is just the total demand in the network, $H$, which is known. Without loosing generality, and for the sake of simplicity, we assume $\hat{C}_{j}=C \forall j$. Since the number of facilities is limited to $p$, we have $H \leq p C$. Then, for a particular value of $p$, we can find the minimum value of $C=H / p$, solve the integer programming formulation, increase the value of $C$, solve again, and keep increasing this value in successive runs of the model. As the value of $C$ increases, representing higher congestion costs, the value of the travel cost objective must either remain the same or decrease (improve), since we are relaxing a constraint of the problem (constraint 13). At some point, constraint (13) is not binding anymore, the solution corresponds to the unconstrained $p$-median solution, and we can stop.

In each solution, although the demand at each facility is bounded above by $C$, it does not necessarily reach this value, so each one of the located facilities can have its particular amount of demand.

Stage 2. For each solution, using the congestion cost function obtained from polls or focus groups, as well as the values of the parameters $K, s$ and $\mu$, compute the waiting cost at each facility. For different values of $K, s$ and $\mu$, different costs will be obtained, and consequently, by changing these values, a calibration of the design can be performed at this stage. Add these costs over all facilities, to obtain the total waiting cost for that solution.

Stage 3. Use the results of the Stages 1 and 2 to draw trade-off curves relating travel and congestion costs. If the cost are in monetary terms, find among all the solutions, the one with the least travel plus congestion cost. Note that the trade-off curves are not necessarily convex nor concave. 


\section{Computational experience on the constraint method}

We solved the model using the constraint method and the linearization, as described above, on a 55-node network (Swain, 1974). The population at each demand node was used as a proxy for the demand rate at that node. The Euclidean distance between nodes was used as a proxy for the travel cost. We assume that the maximum queue length, $K$, at any facility or center, is 15 customers (no matter how many servers there are at that facility). Although we remark that the model accepts different values for each facility, for the example we assume that not only $C$, but the parameters $\mu_{j}$ and $s_{j}$ are the same across the system, omitting the subscript $j$.

Recall that in practice, the queuing cost functions $\left\{b_{n}\right\}$ can be found by polling. In our example, in order to analyze what happens with different types of customers, we use two different possible congestion cost curves, that correspond to customers with different possible perceptions, represented by linear and quadratic cost functions, as shown in Table 3 and Figure 1:

\begin{tabular}{|c|c|c|}
\hline & \multicolumn{2}{|c|}{ Cost functions $\left\{\boldsymbol{b}_{\boldsymbol{n}}\right\}$} \\
\hline $\begin{array}{c}\text { Nr of customers } \\
\text { in queue }\end{array}$ & Linear & Quadratic \\
\hline 0 & 1 & 1 \\
\hline 1 & 1 & 1 \\
\hline 2 & 2 & 4 \\
\hline 3 & 3 & 9 \\
\hline 4 & 4 & 16 \\
\hline 5 & 5 & 25 \\
\hline 6 & 6 & 36 \\
\hline 7 & 7 & 49 \\
\hline 8 & 8 & 64 \\
\hline 9 & 9 & 81 \\
\hline 10 & 10 & 100 \\
\hline 11 & 11 & 121 \\
\hline 12 & 12 & 144 \\
\hline 13 & 13 & 169 \\
\hline 14 & 14 & 196 \\
\hline 15 & 15 & 225 \\
\hline
\end{tabular}

Table 3: Congestion Cost Functions 


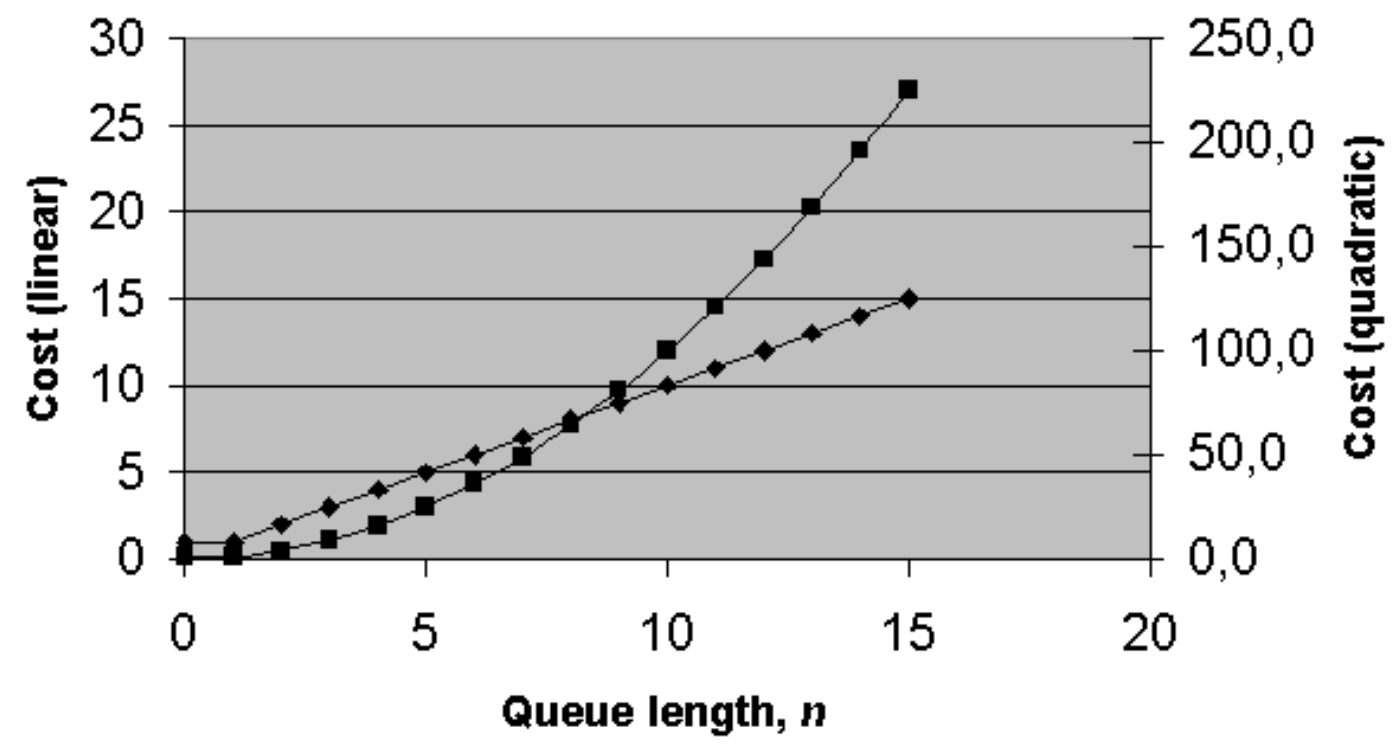

Figure 1: Different cost functions

Since the expected waiting time is proportional to the expected number of customers in queue (both mathematically and intuitively), those customers for which the most important issue is the time spent in queue, will most probably be represented by a linear cost function. The quadratic function, on the other hand, represents the perception of those customers who not only value the time spent in queue, but also dislike crowds or the feeling of standing on line, or in general, those who feel that standing in line is worse than spending time on any other activity. In this case, there is an extra cost that has to be added to the pure time-related cost.

In Stage 1, we solved the optimization model on a Pentium III, $1.6 \mathrm{GHz}, 256 \mathrm{MB}$ RAM computer, running MPL for Windows 4.12 and CPLEX 7.5, for $p=2,3$ and 6 . The sets of solutions for $p=2,3$ and 6 are shown in Tables 4, 5 and 6 . 


\begin{tabular}{|r|r|r|r|}
\hline$C$ & Travel & Demand on & Demand on \\
cost & facility 1 & facility 2 \\
\hline 3200 & 477.505 & 3200 & 3200 \\
\hline 3300 & 476.300 & 3300 & 3100 \\
\hline 3400 & 475.603 & 3390 & 3010 \\
\hline 3500 & 475.554 & 3410 & 2990 \\
\hline 3600 & 474.457 & 3580 & 2820 \\
\hline 3700 & 473.594 & 3700 & 2700 \\
\hline 3800 & 471.968 & 3790 & 2610 \\
\hline 3900 & 471.916 & 3870 & 2530 \\
\hline 4400 & 471.810 & 4360 & 2040 \\
\hline 5000 & 467.427 & 5000 & 1400 \\
\hline 5200 & 461.820 & 5200 & 1200 \\
\hline 5230 & 461.477 & 5230 & 1170 \\
\hline
\end{tabular}

Table 4: Set of solutions for $p=2$

\begin{tabular}{|r|r|r|r|r|}
\hline \multicolumn{1}{|c|}{$C$} & $\begin{array}{c}\text { Travel } \\
\text { cost }\end{array}$ & $\begin{array}{r}\text { Demand on } \\
\text { facility 1 }\end{array}$ & $\begin{array}{r}\text { Demand on } \\
\text { facility 2 }\end{array}$ & $\begin{array}{r}\text { Demand on } \\
\text { facility 3 }\end{array}$ \\
\hline 2140 & 418.123 & 2140 & 2140 & 2120 \\
\hline 2200 & 416.998 & 2190 & 2090 & 2120 \\
\hline 2300 & 414.038 & 2290 & 2300 & 1810 \\
\hline 2400 & 412.716 & 2400 & 2090 & 1910 \\
\hline 2500 & 410.728 & 2500 & 2420 & 1480 \\
\hline 2600 & 405.884 & 2600 & 2600 & 1200 \\
\hline 2700 & 404.477 & 2690 & 2540 & 1170 \\
\hline 2800 & 402.883 & 2640 & 2800 & 960 \\
\hline 2900 & 401.432 & 2550 & 2890 & 960 \\
\hline 3000 & 399.908 & 2450 & 2990 & 960 \\
\hline 3100 & 398.912 & 3080 & 2150 & 1170 \\
\hline 3300 & 398.416 & 3300 & 1930 & 1170 \\
\hline 3400 & 394.788 & 3390 & 1810 & 1200 \\
\hline 3500 & 393.474 & 3470 & 1760 & 1170 \\
\hline 3700 & 390.616 & 3690 & 1540 & 1170 \\
\hline 3900 & 388.283 & 3890 & 1200 & 1310 \\
\hline 4040 & 387.024 & 4040 & 1170 & 1190 \\
\hline & & & & \\
\hline
\end{tabular}

Table 5: Set of solutions for $p=3$ 
For $\mathrm{p}=2$, the run times for the solutions were between 0.6 and $51 \mathrm{sec}$. The number of Branch and Bound nodes ranged between none and 55. For $p=3$, the run times for the solutions were between 0.7 and $112 \mathrm{sec}$. The number of Branch and Bound nodes ranged between none and 255 .

\begin{tabular}{|r|r|r|r|r|r|r|r|}
\hline$C$ & $\begin{array}{c}\text { Travel } \\
\text { cost }\end{array}$ & $\begin{array}{r}\text { Demand on } \\
\text { facility 1 }\end{array}$ & $\begin{array}{r}\text { Demand on } \\
\text { facility 2 }\end{array}$ & $\begin{array}{r}\text { Demand on } \\
\text { facility 3 }\end{array}$ & $\begin{array}{r}\text { Demand on } \\
\text { facility 4 }\end{array}$ & $\begin{array}{r}\text { Demand on } \\
\text { facility 5 }\end{array}$ & $\begin{array}{r}\text { Demand on } \\
\text { facility 6 }\end{array}$ \\
\hline 1090 & 297.84 & 1090 & 1090 & 1080 & 1090 & 980 & 1070 \\
\hline 1100 & 296.88 & 1100 & 1090 & 1080 & 1100 & 960 & 1070 \\
\hline 1200 & 289.127 & 1200 & 1200 & 1200 & 1110 & 1080 & 610 \\
\hline 1300 & 280.11 & 1300 & 1300 & 1300 & 930 & 960 & 610 \\
\hline 1400 & 275.523 & 1400 & 1320 & 1380 & 730 & 960 & 610 \\
\hline 1500 & 273.385 & 1500 & 1260 & 1320 & 750 & 960 & 610 \\
\hline 1600 & 272.165 & 1580 & 1220 & 1300 & 730 & 960 & 610 \\
\hline 2500 & 268.925 & 2500 & 1380 & 930 & 510 & 470 & 610 \\
\hline 2800 & 265.617 & 2800 & 1300 & 730 & 490 & 470 & 610 \\
\hline
\end{tabular}

Table 6: Set of solutions for $p=6$

For $\mathrm{p}=6$, the run times for the solutions were between 0.5 and $147 \mathrm{sec}$. The number of Branch and Bound nodes ranged between none and 774.

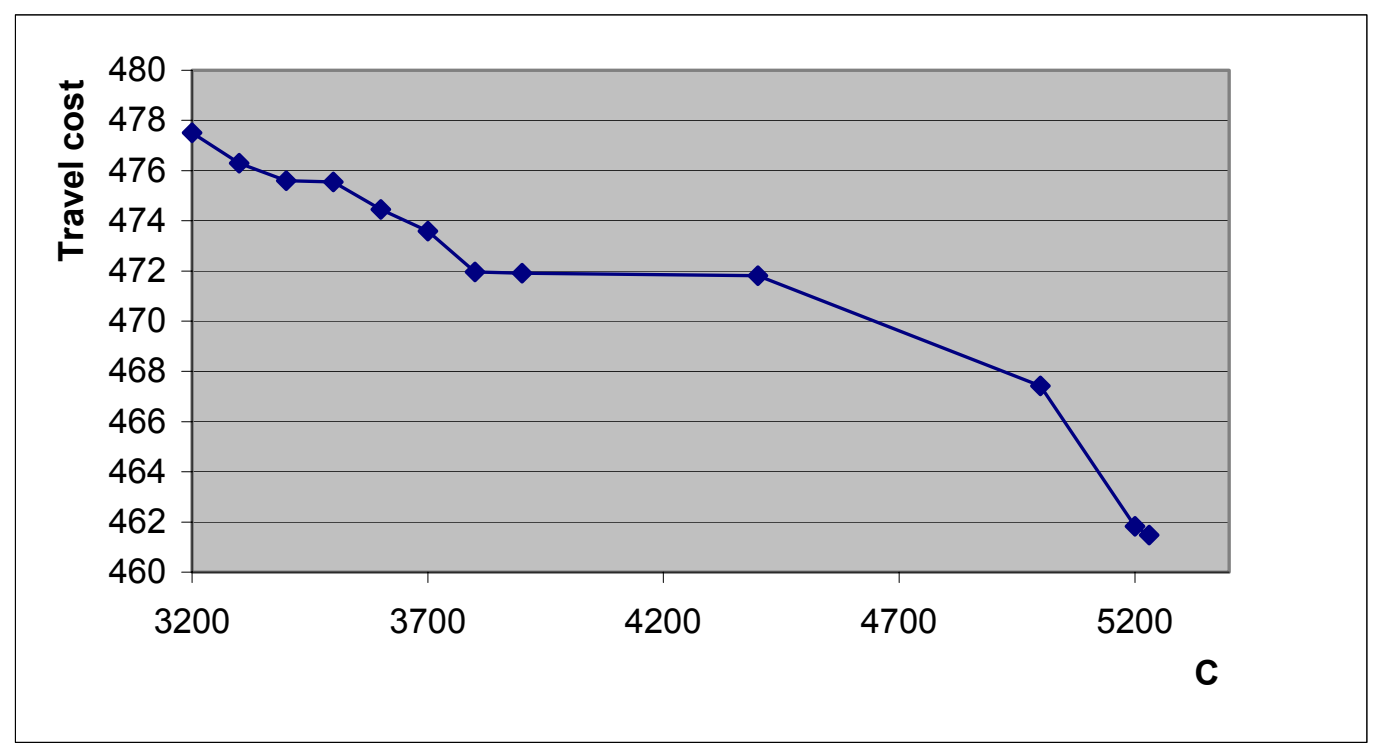

Figure 2: Trade off between travel costs and $C$, for $p=2$. 
For these cases, we computed the trade - off curves between the demand limit, $C$, and the travel cost. The curves are shown in Figures 2, 3 and 4. Note that these curves are neither convex nor concave, due to the integer nature of the problem.

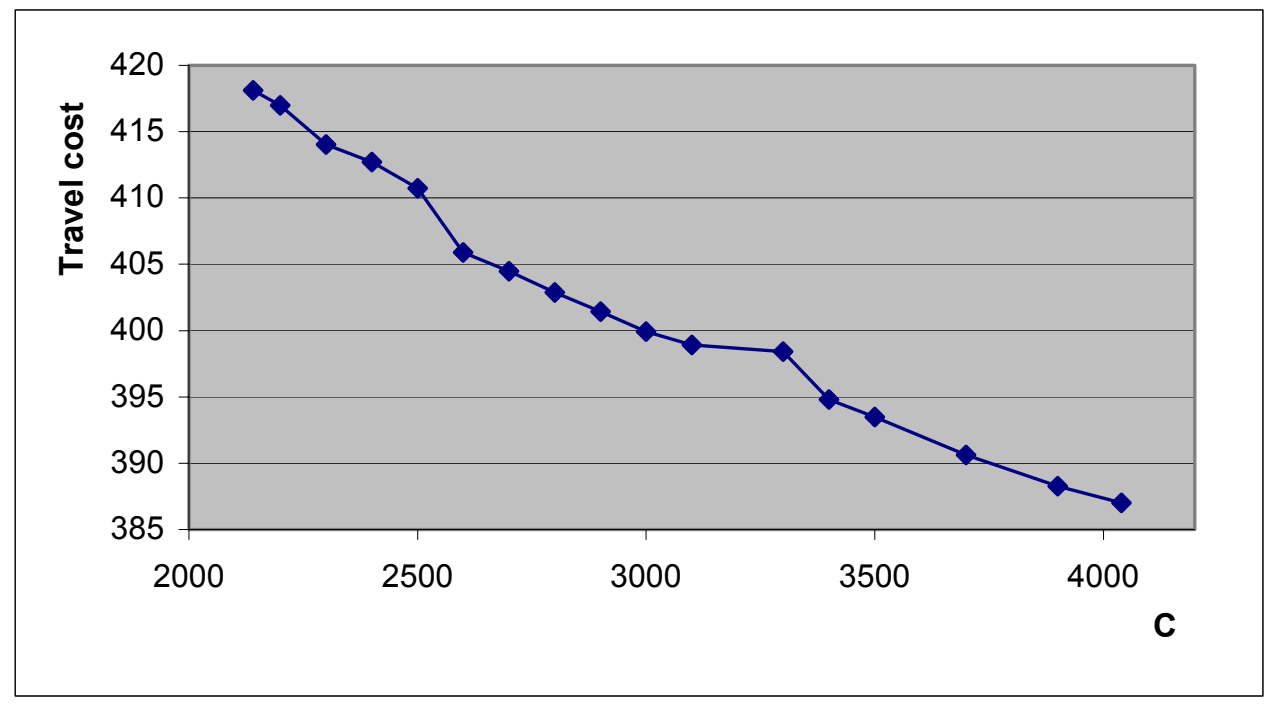

Figure 3: Trade off between travel costs and $C$, for $p=3$.

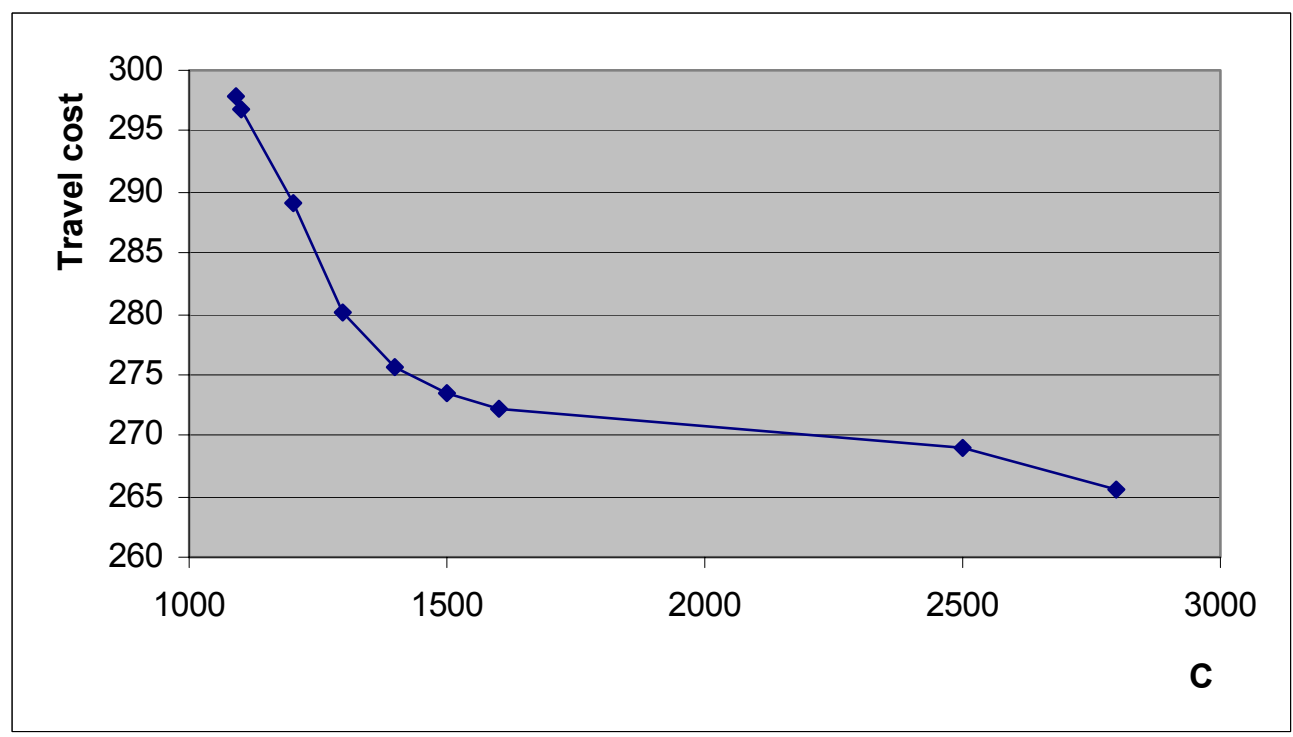

Figure 4: Trade off between travel costs and $C$, for $p=6$. 


\begin{tabular}{|r|r|r|r|r|r|}
\hline & Travel & \multicolumn{2}{c|}{ Linear congestion costs } & \multicolumn{2}{c|}{ Quadratic congestion costs } \\
\cline { 3 - 6 } \multicolumn{1}{r|}{ cost } & facility 1 & facility 2 & facility 1 & facility 2 \\
\hline 3200 & 477.505 & 48.846 & 48.846 & 631.79 & 48.846 \\
\hline 3300 & 476.300 & 51.426 & 46.161 & 673.24 & 46.161 \\
\hline 3400 & 475.603 & 53.67 & 43.643 & 709.28 & 43.643 \\
\hline 3500 & 475.554 & 54.159 & 43.07 & 717.13 & 43.07 \\
\hline 3600 & 474.457 & 58.202 & 37.983 & 781.95 & 37.983 \\
\hline 3700 & 473.594 & 60.95 & 34.167 & 825.85 & 34.167 \\
\hline 3800 & 471.968 & 62.965 & 31.203 & 857.94 & 31.203 \\
\hline 3900 & 471.916 & 64.726 & 28.521 & 885.93 & 28.521 \\
\hline 4400 & 471.810 & 75.089 & 13.468 & 1049 & 107.2 \\
\hline 5000 & 467.427 & 87.983 & 4.1444 & 1249.6 & 15.526 \\
\hline 5200 & 461.820 & 91.93 & 2.9204 & 1310.5 & 8.5929 \\
\hline 5230 & 461.477 & 92.519 & 2.771 & 1319.6 & 7.8715 \\
\hline
\end{tabular}

Table 7: Costs for all solutions, $p=2, \mu=800, s=3$.

\begin{tabular}{|r|r|r|r|r|r|r|r|}
\hline & \multirow{2}{*}{$\begin{array}{c}\text { Travel } \\
C\end{array}$} & \multicolumn{3}{|c|}{ Linear congestion costs } & \multicolumn{3}{c|}{ Quadratic congestion costs } \\
\cline { 3 - 8 } & facility 1 & facility 2 & facility 3 & facility 1 & facility 2 & facility 3 \\
\hline 2140 & 418.123 & 11.71 & 11.71 & 11.24 & 86.47 & 86.47 & 81.6 \\
\hline 2200 & 416.998 & 12.93 & 10.57 & 11.24 & 99.53 & 74.57 & 81.6 \\
\hline 2300 & 414.038 & 15.57 & 15.85 & 5.77 & 128.90 & 132.05 & 29.0 \\
\hline 2400 & 412.716 & 18.72 & 10.57 & 7.18 & 165.40 & 74.57 & 41.4 \\
\hline 2500 & 410.728 & 21.70 & 19.31 & 2.95 & 201.27 & 172.41 & 8.5 \\
\hline 2600 & 405.884 & 24.70 & 24.70 & 1.85 & 238.55 & 238.55 & 3.3 \\
\hline 2700 & 404.477 & 27.37 & 22.90 & 1.77 & 272.49 & 216.08 & 3.0 \\
\hline 2800 & 402.883 & 25.89 & 30.54 & 1.31 & 253.63 & 313.62 & 1.7 \\
\hline 2900 & 401.432 & 23.20 & 33.04 & 1.31 & 219.80 & 346.52 & 1.7 \\
\hline 3000 & 399.908 & 20.20 & 35.70 & 1.31 & 183.09 & 382.01 & 1.7 \\
\hline 3100 & 398.912 & 37.99 & 11.95 & 1.77 & 412.87 & 88.99 & 3.0 \\
\hline 3300 & 398.416 & 43.22 & 7.50 & 1.77 & 484.05 & 44.40 & 3.0 \\
\hline 3400 & 394.788 & 45.22 & 5.77 & 1.85 & 511.56 & 29.02 & 3.3 \\
\hline 3500 & 393.474 & 46.96 & 5.18 & 1.77 & 535.32 & 24.16 & 3.0 \\
\hline 3700 & 390.616 & 51.51 & 3.30 & 1.77 & 597.80 & 10.61 & 3.0 \\
\hline 3900 & 388.283 & 55.44 & 1.85 & 2.20 & 651.65 & 3.27 & 4.7 \\
\hline 4040 & 387.024 & 58.30 & 1.77 & 1.83 & 690.61 & 2.98 & 3.2 \\
\hline
\end{tabular}

Table 8: Costs for all solutions, $p=3, \mu=800, s=3$. 


\begin{tabular}{|c|r|r|r|r|r|r|r|}
\hline & Travel & \multicolumn{7}{|c|}{ Quadratic congestion costs } \\
\cline { 3 - 8 }$C$ & cost & Facility 1 & Facility 2 & Facility 3 & Facility 4 & Facility 5 & Facility 6 \\
\hline 1090 & 297.84 & 17.40 & 17.40 & 16.56 & 17.40 & 10.1 & 15.75 \\
\hline 1100 & 296.88 & 18.30 & 17.40 & 16.56 & 18.30 & 9.2 & 15.75 \\
\hline 1200 & 289.127 & 30.15 & 30.15 & 30.15 & 19.23 & 16.6 & 1.86 \\
\hline 1300 & 280.11 & 48.99 & 48.99 & 48.99 & 7.94 & 9.2 & 1.86 \\
\hline 1400 & 275.523 & 76.87 & 53.80 & 70.51 & 3.16 & 9.2 & 1.86 \\
\hline 1500 & 273.385 & 114.41 & 40.47 & 53.80 & 3.45 & 9.2 & 1.86 \\
\hline 1600 & 272.165 & 150.43 & 33.28 & 48.99 & 3.16 & 9.2 & 1.86 \\
\hline 2500 & 268.925 & 562.31 & 70.51 & 7.94 & 1.21 & 1.0 & 1.86 \\
\hline 2800 & 265.617 & 664.73 & 48.99 & 3.16 & 1.10 & 1.0 & 1.86 \\
\hline
\end{tabular}

Table 9: Quadratic costs for all solutions, $p=6, \mu=800, s=2$.

In the example network, $\sum_{i} h_{i}=H=6,400$, so for two facilities the minimum possible value of $C$ is 3,200 . Below this value, the problem becomes infeasible. The $p$-median solution corresponds to $C=5,230$.

In Stage 2, we computed the waiting cost of each solution, for linear and quadratic congestion cost curves. For $p=6$, only the quadratic costs were computed.

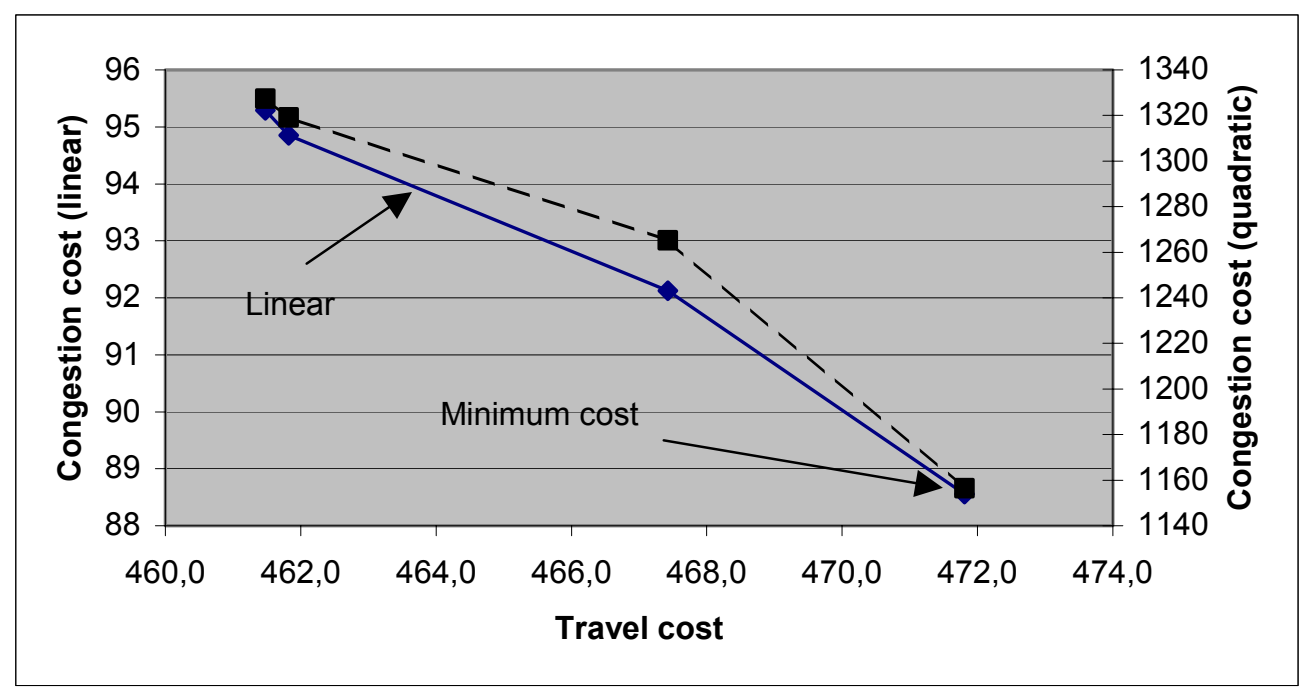

Figure 5: Trade off between travel and congestion costs, for $p=2$. 


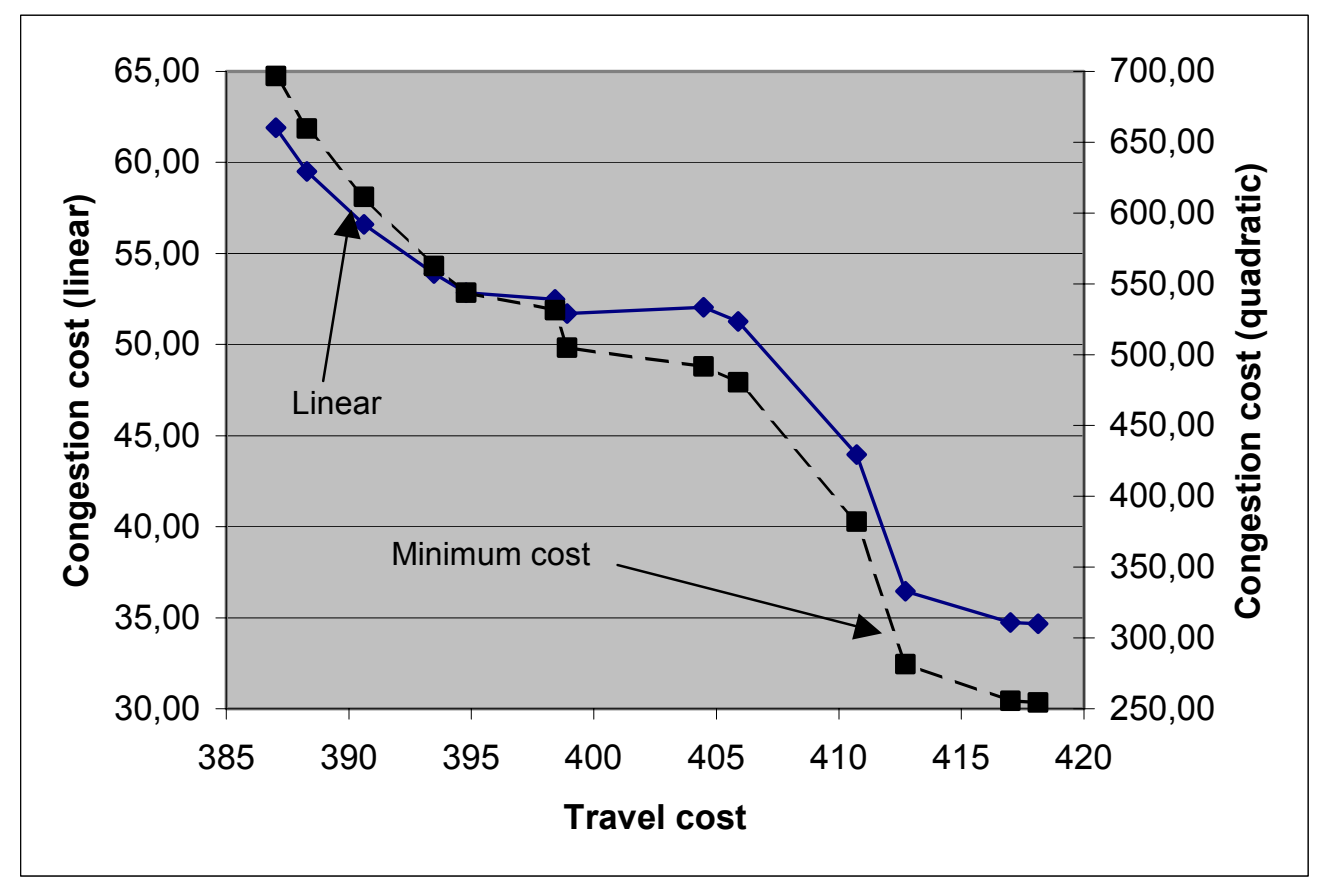

Figure 6: Trade off between travel and congestion costs, for $p=3$.

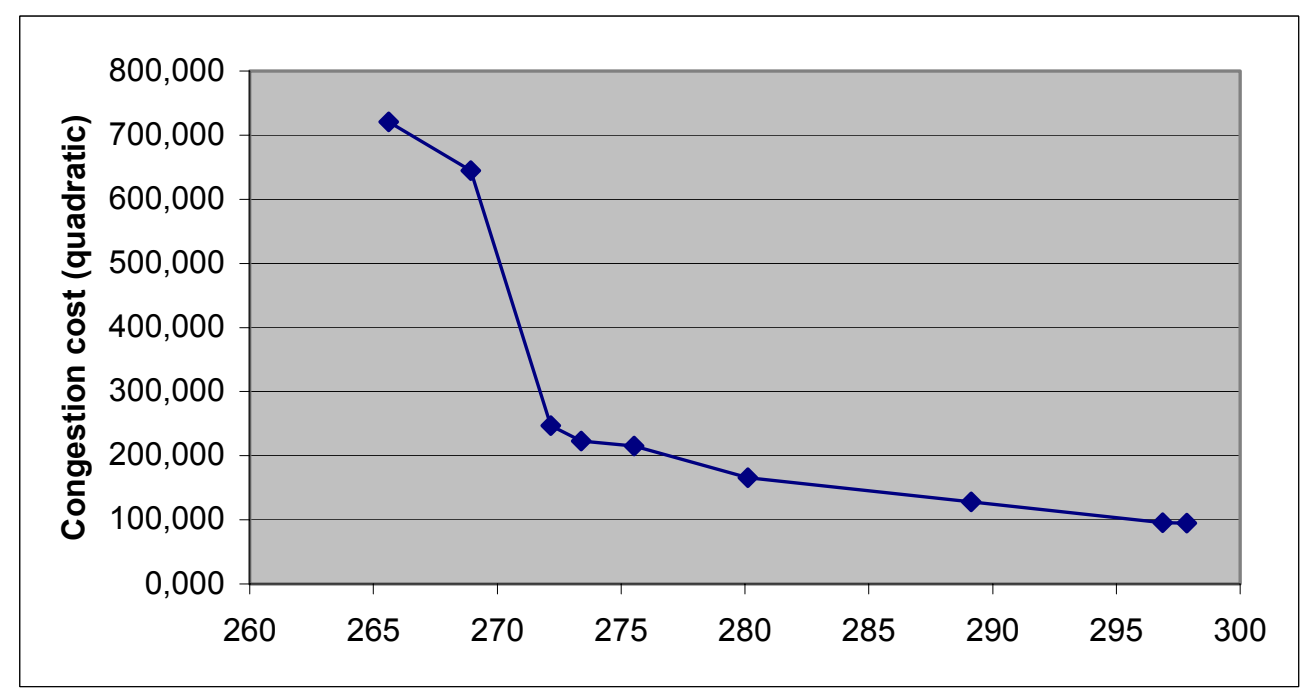

Figure 7: Trade off between travel and congestion costs, for $p=6$. 


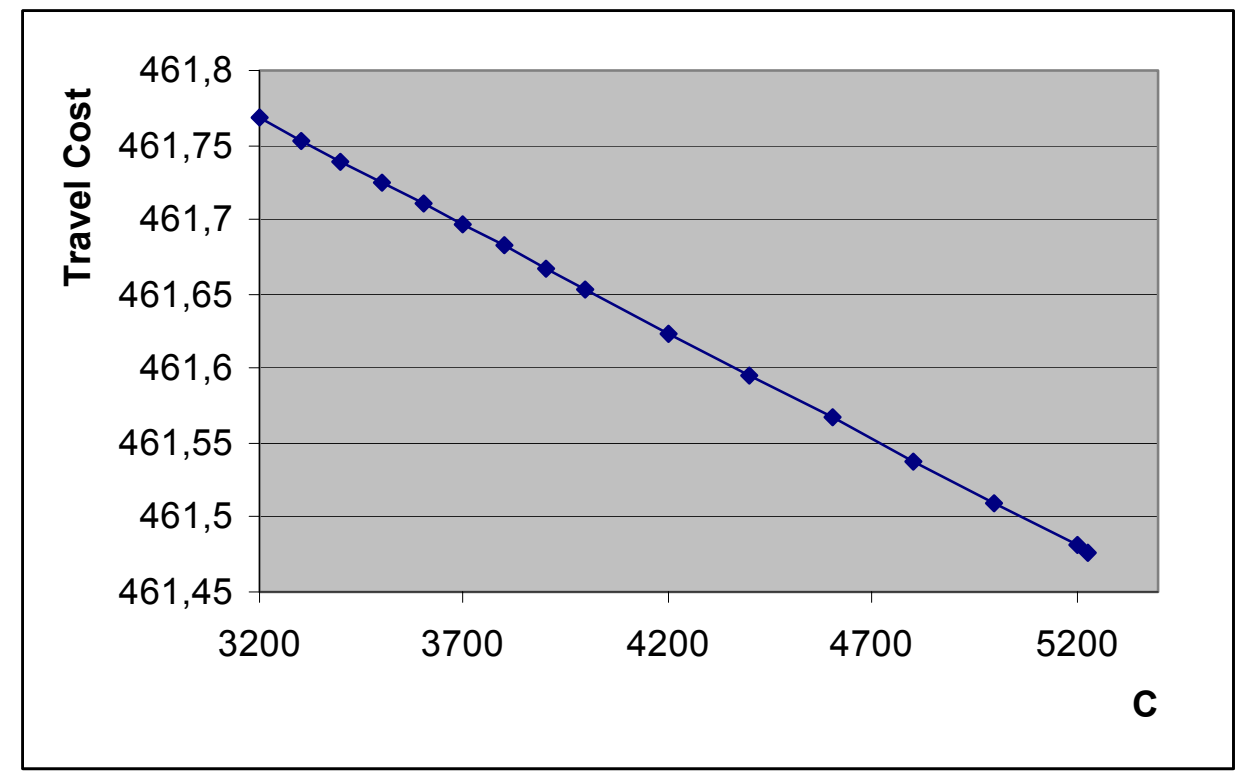

Figure 8: Congestion cost curve for $\mu=800, s=3$

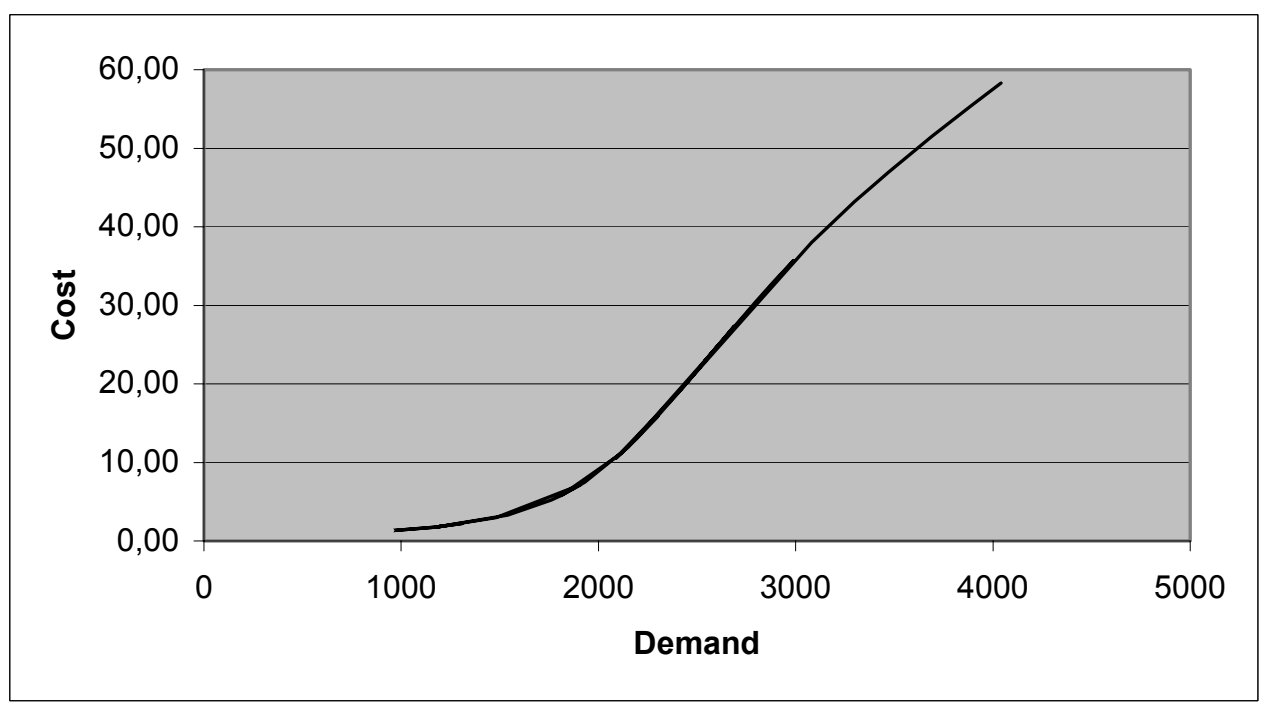

Figure 9: Travel cost trade-off 
Note that the number of servers per center is different in the cost computation for $p=6$. The maximum queue length, $K$, was set to 15 for all cases. In each case, the costs for the linear case and for the quadratic case were added, in order to compute the total congestion cost for that particular solution.

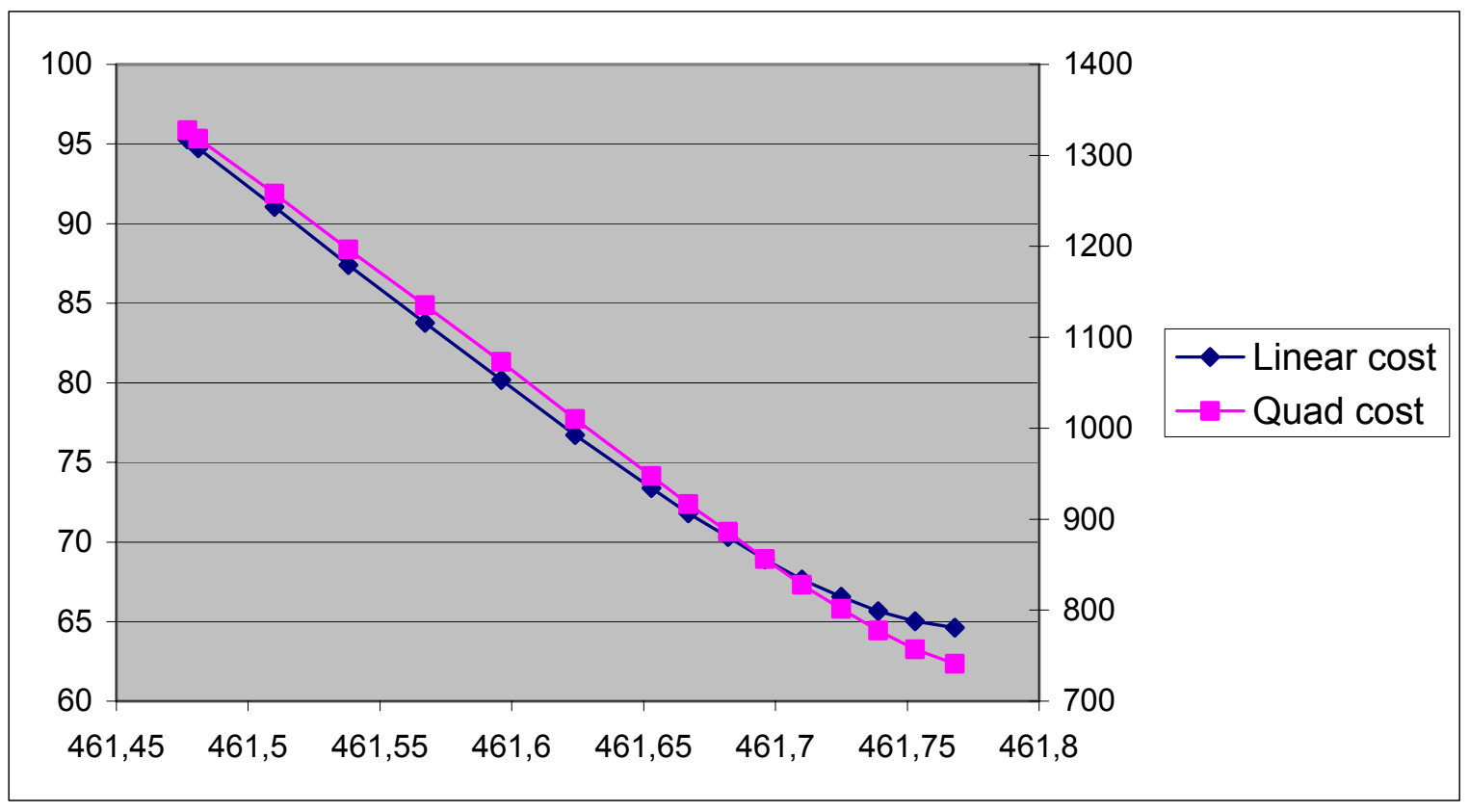

Figure 10: Trade off between travel and congestion costs, for $p=2$, linear relaxation.

As a result of Stage 3 of the procedure, we show on Figures 5 to 7, the trade-off curves, in which only the non-dominated or Pareto optimal solutions are shown.

As an example of computation of the optimal solution when both costs are cast in monetary terms, in Figures 5 and 6, the solutions corresponding to the least total cost are marked with an arrow. Note that the shapes of all the trade-off curves are a result mainly of the integer nature of the variables, enhanced by the neither concave nor convex congestion costs. For example, part of the congestion cost curve for $\mu=800, s=3$ is shown in figure 8 .

As an example of the effect of the integer nature of the variables on the shape of the tradeoff curves, Figures 9 and 10 show the trade-off curves for the linear relaxation of the problem, with $p=2$ :

As expected, the shapes of the trade-off curves of the relaxed versions of the problem are closer to the usual shapes. 


\section{Conclusions}

We propose a model that locates a fixed number of multiple-server service centers or public facilities, both susceptible of becoming congested. Consumers' perceived congestion and travel costs are minimized, considering that all the demand must be served. The travel cost is a general function of the origin and destination of the demand, while the congestion cost is a general function of the number of customers in queue at the facilities, which is proportional to the waiting time. Since the model is nonlinear, we investigate two different solution methods, useful under different circumstances. The first one is based on a MaxMin Ant System and Tabu Search, and finds a solution (not necessarily optimal) in a short time, particularly if the objectives can be easily expressed in the same units. The second procedure is somewhat slower, but allows solving the problem when the relative weights on the objectives are not known, and also allows a deeper analysis of different solutions, particularly if different numbers of servers can be located at different centers. Computational experience is presented on both solution procedures, using in each case such instances of the problem, as to allow a better evaluation of both methods. 


\section{APPENDIX 1: Metaheuristic for the multiobjective nonlinear formulation}

Notation:

- LOCP is the set of center locations.

- $Z_{1}$ is the value of the travel cost objective and $Z_{2}$ is the value of the congestion cost objective

- $\mathrm{W}_{1}$ and $\mathrm{W}_{2}$ are the preset weights on the objectives, that is, the weighted objective $\mathrm{Z}$ is computed as $\mathrm{Z}=\mathrm{W}_{1} \mathrm{Z}_{1}+\mathrm{W}_{2} \mathrm{Z}_{2}$

\section{Procedure:}

1. Initialize the parameters of the Ant System and compute the initial pheromone trail

$$
\tau_{j}=\frac{1}{\sum_{i} h_{i} c_{i j}}
$$

for all nodes $j$ of the network. Make $k=0$.

2. Let $k=k+1$ (iterations of the Ant System procedure)

First Step (FS): Construct a good initial solution

FS.1. Compute $p_{j}=\frac{\tau_{j}}{\tau_{\tau}}$, for all nodes $\mathrm{j}$ of the network. Make $i=0$.

$$
\sum_{l \in J} \tau_{l}
$$

FS.2. Let $i=i+1$

FS.3 Choose randomly a node to locate a center, using the probability function defined in step FS.1.

FS.4 If $i<p$, go to step FS.2.

FS.5 Compute both objectives and find the weighted objective $\mathrm{Z}$

Second Step (SS): Local search phase; Tabu Search.

SS.1. Let $s=0$ (number of times, the diversification criteria is applied).

SS.2. Let $t=0$ (number of iterations of the TABU procedure).

SS.3. Set $Z^{0}($ LOCP $)=Z^{\text {BEST }}$ (LOCP), the best solution found in MAX-MIN Ant system. Set $\mathrm{LOCP}_{\mathrm{i}}{ }^{0}$, for $i=1, \ldots, p$ the optimal locations found in MAX-MIN Ant system.

SS.4. LOCP $_{\mathrm{i}}{ }^{0} \rightarrow \mathrm{LOCP}_{\mathrm{i}+1}{ }^{0}$ (for each located node).

SS.5. Consider all neighborhood nodes $j$ of optimal location node $i$ (i.e. $\left.\operatorname{LOCP}_{\text {ngh } \mathrm{j}\left(\operatorname{Locp}_{\mathrm{i}}^{0}\right)}\right)$ ). Let $\operatorname{LOCP}_{\text {ngh } \mathrm{j}\left(\operatorname{Locp}_{\mathrm{i}}^{0}\right)} \rightarrow \mathrm{LOCP}_{\text {ngh } \mathrm{j}+1\left(\operatorname{Locp}_{\mathrm{i}}^{0}\right)}$. Exchange the facility from node $\mathrm{LOCP}_{\mathrm{i}}^{0}$ $\in$ LOCP to a neighborhood node $\operatorname{LOCP}_{\text {ngh } \mathrm{j}\left(\operatorname{Locp}_{\mathrm{i}}^{0}\right)} \notin \mathrm{LOCP}$.

Check the threshold constraint in this new solution: 
- If this is satisfied, compute the objective function $Z\left(\operatorname{LOCP}_{\left.{\text {ngh } j\left(\operatorname{Locp}_{\mathrm{i}}^{0}\right)}\right)}\right)$.

- If this is not satisfied, set $\mathrm{Z}\left(\operatorname{LOCP}_{\text {ngh }\left(\operatorname{Locpp}_{\mathrm{i}}^{0}\right)}\right)=0$.

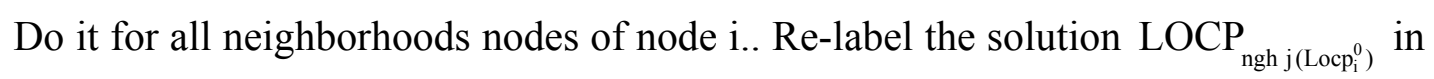
decreasing order of $\mathrm{Z}\left(\mathrm{LOCP}_{\mathrm{ngh} j\left(\operatorname{Locp}_{\mathrm{i}}^{0}\right)}\right)$. When all neighborhoods are visited, go to SS.7

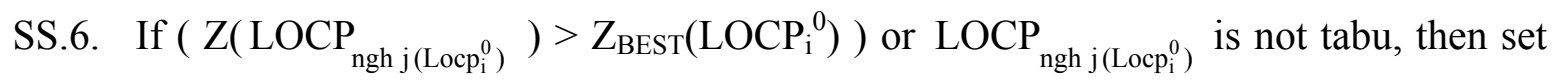
$\mathrm{Z}_{\mathrm{BEST}}\left(\operatorname{LOCP}_{\mathrm{i}}{ }^{0}\right)=\mathrm{Z}\left(\operatorname{LOCP}_{\text {ngh } j\left(\operatorname{Locpp}_{\mathrm{i}}^{0}\right)}\right)$, the outlet is located in $\operatorname{LOCP}_{\text {ngh } j\left(\operatorname{Locpp}_{\mathrm{i}}^{0}\right)}$ and $\mathrm{LOCP}_{\mathrm{i}}{ }^{0}$ is declared tabu until $\mathrm{t}+\varepsilon$, where $\varepsilon$ is a pre-fixed value, and go to step SS.5.

If all nodes visited are tabu and none improves the objective, then the model chooses the node with the lowest tabu tag $(\mathrm{t}+\varepsilon)$ and lift the tabu status of LOCP $\left._{\text {ngh } j\left(\text { Locpo }_{\mathrm{i}}^{0}\right)}\right)$. Then go to step SS.5.

SS.7. If $t$ is less than a pre-fixed upper bound $\mathrm{T}$; update the best solution found $\mathrm{Z}_{\mathrm{BEST}}(\mathrm{LOCP})$, let $\mathrm{t} \rightarrow \mathrm{t}+1$ and go to step SS.3.

8. If $\mathrm{s}<\mathrm{s}^{\mathrm{MAX}}$ (maximum number of times, the diversification criteria is applied). The model starts a new procedure with an initial solution equal to the NP least visited nodes. Let $\mathrm{s}=\mathrm{s}+1$ and go to step SS.2.

Third Step (TS): Update pheromone trails, using the current solution.

TS.1. Compute the news $\tau_{j}$ for all nodes $\mathrm{j}$ of the network, using the optimal locations found in the previous steps. Check also the max $-\min$ limits for all $\tau_{j}$ (i.e. $\left.\tau_{\min } \leq \tau_{j} \leq \tau_{\max }, \forall j\right)$.

TS.2. If $k<$ MAXITER, set $k \rightarrow k+1$ and go to step 2 of the initial procedure. Updating the Best Solution found $\mathrm{Z}_{\mathrm{BEST}}(\mathrm{LOCP})$ in each MAX-MIN Ant system iteration.

Comments about the meta-heuristic:

- In step 6 of Tabu Search, tabu status can be canceled if this permits an improvement in the objective. This rule is called the aspiration criterion

- Step 8 of Tabu Search, states the diversification criterion, that allows a broader exploration of the solution space by starting from locations that have been less well explored. 


\section{Aknowledgements}

This research has been possible thanks to research grants by FONDECYT (Project Nr 1020778, Chile) and by MCYT (Project BEC2000-1027, Spain)

\section{References}

Benati, S and Laporte, G. "Tabu Search algorithms for the $\left(r \mid X_{p}\right)$ - Medianoid and $(r \mid p)$-Centroid Problems". Location Science, 2(4), 1994, pp. 193-204.

Brandeau M., Chiu S., Kumar S., Grossman T., 1995, "Location with Market Externalities" In Facility Location: A Survey of Applications and Methods, Zvi Drezner (ed), 570 pp., Springer Verlag, New York NY. Cohon J., 1978: Multiobjective Programming and Planning, Academic Press, Inc.

Colorni, A. Dorigo, M. and Maniezzo, V. "Distributed optimisation by Ant Colonies", Proceeding of ECAL91 - European Conference on Artificial Life: Elsevier Publishing, Paris, France, 1991a, pp. 134-142.

Colorni, A. Dorigo, M. and Maniezzo, V. "The Ant System: Optimisation by a Colony of Cooperating Agents", IEEE Transactions on Systems, Man and Cybernetics - Part B, 26(1), 1991b, pp. 29-41.

Dorigo, D. and DiCaro, G. "The ant colony optimization meta-heuristic". In D.Corne, M.Dorigo and F.Glover (eds), News Ideas in Optimisation, 1999, McGraw-Hill.

Gendreau, M.; Hertz, Z. and Laporte, G. "A Tabu Search Heuristic for the Vehicle Routing Problem". Management Science 40(10), 1994, pp.1276-1289.

Glover. F. "Tabu Search, part I“. ORSA Journal of Computing, 1, 1989, pp.190-206

Glover. F. "Tabu Search, part II". ORSA Journal of Computing, 2:, 1990, pp. 4-32.

Hillier F S, and Lieberman G J, 1986, Introduction to Operations Research, Holden-Day, Inc., Oakland, CA.

Jamil M, Baveja A, Batta R, 1999, "The Stochastic Queue Center Problem", Computers and Operations Research, 26, 1423-1436.

Klincewicz, J.G. "Avoiding Local Optima in the p-Hub location problem using Tabu Search and GRASP". Annals of Operations Research, 40, 1992, pp. 283-302

Lourenço, H. and Serra, D. "Meta-heuristics for the Generalized Assignment Problem". In Mathware \& Soft Computing, Special Issue on Ant Colony Optimization: Models and Applications, forthcoming

Marianov V., Serra D., 1998, "Probabilistic Maximal Covering Location-Allocation for Congested Systems", Journal of Regional Science, 38 (3), 401 - 424.

Marianov V., Serra D., 2001, "Hierarchical Location-Allocation models for Congested Systems", European Journal of Operational Research, 135(1), $196-209$.

Marianov V., Serra D., ReVelle C., 1999, "Location of hubs in a competitive environment" - European Journal of Operations Research, 114, 363 - 371.

Rolland E, Amiri A, Barkhi R, 1999: "Queuing Delay Guarantees in Bandwith Packing", Computers and Operations Research, 26, 921 - 935.

Rolland, E.; Schilling, D.A. and Current, J.R. "An efficient Tabu Search procedure for the p-Median problem“. European Journal of Operation Research 96(2), 1997, pp. 329-342.

Stüzle, T. "An ant approach for the flow shop problem". In proceeding of the $6^{\text {th }}$ European Congress on Intelligent Techniques \& Soft Computing (EUFIT'98), 3, 1998, pp. 1560-1564.

Stüzle, T. "Local Search Algorithms for Combinatorial Problems - Analysis, Improvements, and New Applications". PhD thesis, Department of Computer Science, Darmstadt University of Technology, Germany, 1998.

Stüzle, T. "MAX-MIN Ant System for the Quadratic Assignment Problem". Technical Report AIDA-97-4, 1997, FG Intellektik, TU Darmstadt, Germany. 
Stüzle, T. and Hoos, H. "Max-Min Ant System and Local Search for Combinatorial Optimisation", in S.Voß, S. Martello, I.H. Osman and C. Roucairol (eds.), Meta-Heuristics: Trends in Local Search paradigms for Optimisation, 1999, Kluwer Academic Publishers, pp. 313-329. 